Article original

\title{
Facteurs de risque et mortalité associés aux infections nosocomiales en unité de soins intensifs : étude cas-témoins
}

\author{
Risk factors and mortality associated with nosocomial infections in intensive care \\ unit: a case-control study
}

\author{
Abdessamad Dali Ali 1 , Houaria Beldjilali 1, Fouzia Aggag 1, Mohamed Zina 4, Salah Ouhadj 1, Radia Dali \\ Yahia 2, Khadidja Meddeber 3, Nori Midoun 1 \\ 1.Service d'épidémiologie ,établissement hospitalo universitaire 1er novembre d'Oran ,Faculté de médecine, université Oran1 \\ 2.Service de microbiologie, établissement hospitalo universitaire 1er novembre d'Oran ,Faculté de médecine, université Oran1 \\ 3.EHS Canastel- 4.Etablissement public de santé de proximité de Boutlelis,Oran
}

Auteur correspondant: daliali.abdessamad@yahoo.com Soumis le 11/05/2020; accepté le 01/07/2020 ; publié le 21/12/2020

Citation:DALI ALI,A.,et al. Facteurs de risque et mortalité associés aux infections nosocomiales en unité de soins intensifs : étude cas-témoins.(2020) J Fac Med Or 4(2):587-596.

DOI:10.5281/zenodo.4282446

\section{MOTS CLÉS}

Facteurs pronostiques, infections associées aux soins, mortalité attribuable

\section{Résumé}

Introduction-Les infections nosocomiales (IN) représentent un véritable problème de santé publique, en particulier dans les unités de soins intensifs, dans lesquelles les risques d'IN et de décès sont plus élevés, essentiellement lorsque ces infections sont dues à des bactéries multirésistantes.

Objectif- Déterminer les principaux facteurs associés, aussi bien à la survenue d'IN qu'à la survenue de décès en réanimation.

Méthodes-Il s'agissait d'une étude cas-témoins non appariée, nichée dans la cohorte des patients admis dans l'unité de réanimation adulte de l'établissement hospitalier et universitaire d'Oran (Algérie), du 3 juin 2012 au 31 décembre 2013. Les cas $(n=73)$ correspondaient aux patients ayant développé une IN et les témoins $(n=232)$ à ceux qui en étaient indemnes. Les facteurs de risque étudiés correspondaient aux expositions aux différents dispositifs médicaux invasifs. Pour étudier la relation entre les différents types de variables, le test du Chi-deux a été utilisé pour les variables catégoriques et le test de Student pour les variables quantitatives.Le seuil de signification statistique $p$ a été fixé à $5 \%$.

Résultats-En analyse multifactorielle, le sexe masculin (ORa $=3,16, p=0,03)$ et la durée de cathétérisme veineux central supérieure à 8 jours $(\mathrm{RCa}=4,7, \mathrm{p}=0,01)$ étaient significativement associés à la survenue d'infection associée aux soins. De même, la survenue de décès était associée à trois facteurs pronostiques, à savoir : un âge supérieur à 65 ans $(R C a=4,31, p<0,01)$, la présence d'intubation $(R C a=9.49$, $p<0,001)$ et la présence de cathétérisme veineux central ( $R C a: 2,43, p=0,02$ ). L'IN, quelle que soit sa localisation, n'était pas associée à la survenue de décès.

Conclusion-L'exposition prolongée aux différents dispositifs invasifs représentait le principal facteur de risque lié à la survenue d'IN en milieu de réanimation. Cependant, aucune association significative n'a été trouvée entre l'IN et la survenue de décès. 


\section{KEY WORDS}

Attributable morta-
lity,health care associated
infections,prognostic
factors

\section{I.Introduction}

Les infections nosocomiales (IN) représentent un véritable problème de santé publique, en particulier dans les unités de soins intensifs [1]où l'exposition aux différents dispositifs médicaux invasifs (DMI) serait responsable de 60\% de ces infections [2]. En effet, selon une étude multicentrique[1], ayant concerné 703 unités de soins intensifs, réparties dans 50 pays, la densité d'incidence des pneumonies acquises sous ventilation mécanique (PAVM) était 13,1 pour 1000 jours d'intubation. De plus, les densités d'incidence des infections urinaires liées au sondage (IUS) et des infections liées au cathétérisme veineux central (ILC) étaient, respectivement, de 5,07 IUS pour 1000 jours de sondage urinaire et de 4,1 ILC pour 1000 jours de cathétérisme veineux central (CVC). En France, l'évolution des ILC a connu une nette diminution, en passant de 1,56\%, en 2004, à 0,59\%, en 2016. Par contre, le taux d'incidence des bactériémies nosocomiale est restée, presque, le même durant cette même période, avec des taux compris entre 3,41\% et 3,98\%[3]. La survenue d'IN liée aux DMI n'est pas sans conséquence sur le pronostic vital. En effet, la mortalité attribuable aux PAVM peut varier de 0 à 50\%[4]. De même, la survenue d'IUS serait associée à l'augmentation de la mortalité brute[5], quoique la mortalité attribuable à cette dernière soit toujours controversée[6]. De plus, la mortalité attribuable aux bactériémies nosocomiales secondaires aux ILC variait de $4 \%$ à $25 \%$ selon la méta-analyse de Siemposet al. [7]. Les disparités constatées entre les études, en matière de mortalité, peuvent être dues à l'hétérogénéité des pathologies dont ces patients souffrent, ainsi qu'à la sévérité de leur état. De plus, la non-prise en compte de certains facteurs confondants, en particulier l'exposition aux DMI, peut éventuellement expliquer certaines dissimilitudes. Au meilleures des connaissances des auteurs, aucune étude Algérienne antérieure n'a identifié les facteurs de risque associés à la survenue d'IN en réanimation ou n'a estimé la mortalité attribuable à cette IN. De ce fait, et partant de l'hypothèse que l'exposition aux DMI pourrait être associée aussi bien à la survenue d'IN qu'à la survenue de décès, l'objectif de cette étude était d'identifier les principaux facteurs de risque associés à la survenue d'IN et d'estimer la mortalité attribuable à cette dernière en réanimation.

\section{Patients et méthodes}

\section{Type d'étude}

Il s'agissait d'une étude de type cas-témoins non appariée, nichée dans la cohorte des patients admis dans l'unité de réanimation adulte des urgences médico-chirurgicales de l'Etablisse- 
ment Hospitalier et Universitaire d'Oran (EHUO), du 3 juin 2012 au 31 décembre 2013. Cette unité comportait 10 lits de réanimation avec l'hospitalisation, en moyenne, de 230 patients par an. Ces derniers étaient, le plus souvent, admis dans le cadre d'un polytraumatisme secondaire à un accident de la voie publique.

\section{Aspects éthiques}

Les principes de la déclaration d'Helsinki étaient respectés en ce qui concerne, la protection de la vie privée et la confidentialité des informations personnelles.

\section{Recueil de données}

3.1.Données de séjour hospitalier-Ils s'agissait des dates d'entrée dans le service, de sortie ou de décès.

3.2. Facteurs de risque-Les facteurs suivants étaient renseignés, à savoir : l'âge, le sexe, le traitement antibiotique à l'admission, la notion de traumatisme, la présence d'immunodépression, le mode d'admission (évacuation ou transfert d'un autre service), et la notion de chirurgie.

\subsection{Exposition aux dispositifs invasifs}

L'évaluation de l'exposition aux différents DMI invasifs avait concerné l'intubation et/ou la trachéotomie, le sondage urinaire, et le CVC. Les dates de mise en place et de retrait de chaque dispositif invasif étaient mentionnées sur les fiches d'enquêtes afin de calculer la durée d'exposition à chaque DMI.

\section{Population de l'étude}

Figure 1. Description du protocole d'enquête

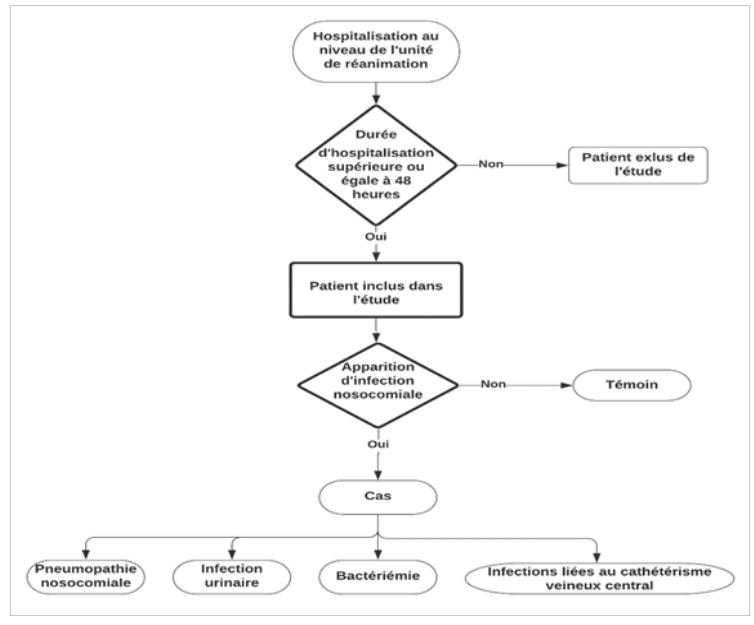

4.1. Cas

\subsubsection{Critères d'inclusion}

Comme le montre la figure 1, tous les patients ayant développé l'une des IN suivantes étaient inclus dans l'étude, à savoir les pneumopathies nosocomiales, les infections urinaires, les ILC et les bactériémies. Les définitions utilisées étaient celles des centres de contrôle et de prévention des maladies et du réseau national de sécurité sanitaire des États Unis [8]. Seules les IN survenues 48 heures, au moins, après hospitalisation étaient incluses dans l'étude.

\subsubsection{Pneumopathie nosocomiale}

La pneumopathie nosocomiale était définie par [8] : la présence de deux clichés radiologiques ou plus avec une image évocatrice de pneumonie.

-Et une hyperthermie $>38^{\circ} \mathrm{C}$ sans autre cause et une leucopénie < 4000 éléments $/ \mathrm{mm} 3$ ou une hyperleucocytose > 12000 éléments/mm3.

-Et au moins, un des signes suivants : apparition de sécrétions purulentes ou modifications des caractéristiques (couleur, odeur, quantité, consistance), toux ou dyspnée ou tachypnée, présence de râles crépitants à l'auscultation, aggravation des gaz du sang [pression artérielle partielle en oxygène ( $\mathrm{PaO2}) /$ la fraction inspirée d'02(FiO2) $\leq 240$ ]ou besoins accrus en oxygène ou en assistance respiratoire.

-Et un prélèvement distal protégé avec un seuil de 103 unités formant colonie (UFC)/ml.

\subsubsection{Infection urinaire}

Cliniquement, l'infection urinaire était définit[8]par la présence, d'au moins, un des signes suivants : fièvre supérieure à $38^{\circ} \mathrm{C}$, impériosité mictionnelle, pollakiurie, brûlure mictionnelle, et douleur sus-pubienne (en l'absence d'autre cause, infectieuse ou non). Les critères de diagnostic différaient selon la présence ou non de sonde urinaire. Ainsi, en absence de sondage vésical, le diagnostic de l'infection urinaire reposait sur la présence d'une leucocyturie supérieure ou égale à 104 éléments/ml et d'une uroculture positive supérieure ou égale à 103 micro-organismes/ $\mathrm{ml}$ avec, au plus, l'isolement deux micro-organismes différents. Par contre, en présence de sondage vésical en cours ou dans les 7 jours précédents, le diagnostic d'infection urinaire reposait sur la présence d'uroculture positive supérieure ou égale à 105 micro-organismes/ml avec, au plus, l'identification de deux micro-organismes différents.

\subsubsection{Infection liée au cathétérisme veineux central}

Le diagnostic d'ILC [8] reposait sur la présence d'une culture quantitative du CVC supérieure ou égale à $103 \mathrm{UFC} / \mathrm{ml}$ et la purulence de l'orifice d'entrée du cathéter.

\subsubsection{Bactériémie nosocomiale}

La bactériémie nosocomiale était définie [8]par la présence d'une hémoculture positive, au moins, justifiée par des signes cliniques.

\subsubsection{Critères d'exclusion}

- Toute IN importée d'un autre service ou d'un autre établissement.

- Les autres types d'IN,y compris les infections du site opéra- 
toire.

\subsection{Témoins}

Il s'agit des patients ayant été hospitalisés pendant au moins 48 heures et n'ayant pas développé d'IN au cours de leur hospitalisation.

\section{Calcul du nombre de sujets nécessaires}

L'exposition aux différents DMl serait responsable de la survenue d'infections nosocomiales en réanimation. De ce fait, le nombre de sujets nécessaires est calculé en se basant sur le pourcentage d'exposition à, au moins, un DMI, parmi les cas et les témoins, selon la formule proposée par Kelsey et al. [9].

$\left.\left.n 1=\left(Z_{\infty} / 2+Z 1-B\right)\right) 2 p q(r+1)\right) / r(p 1-p 2) 2$ et $n 2=r n 1$

où $\mathrm{n} 1$ est le nombre de cas, n2 le nombre de témoins, $Z_{\infty} / 2=$ 1,96 (pour un risque d'erreur de $5 \%$ ), Z1-B $=0,842$ (pour une puissance de $80 \%$ ), p1 proportion de cas exposés $(98,76 \%)[10]$, p2 proportion de témoins exposés (86.41\%) [10].

Le petit $r$ fait référence au nombre de témoins par cas. Dans cette étude, il est égal à 3. De même, $p$ correspond à (p1+rp2)/ $(\mathrm{r}+1)$ et $\mathrm{q}$ correspond à $1-\mathrm{p}$.

Après application de la formule, le nombre de sujets nécessaires, permettant de mettre en évidence une différence significative entre les cas et les témoins, est le suivant : $\mathrm{n} 1=65$, n2 = 194, ce qui correspond à 259 patients au total.

\section{Analyse des données}

La saisie et l'analyse unifactorielle des données étaient réalisées à l'aide des logiciels Epi-Info 7et Open-Epi du CDC d'Atlanta. Le test du Chi-deux était utilisé pour les variables catégoriques et le test de Student pour les variables quantitatives. Le seuil de signification statistique $\mathrm{p}$ a été fixé à $5 \%$. Les tableaux à double contingence étaient établis pour le calcul du Rapport de Cotes (RC)comme facteur d'association épidémiologique avec établissement des intervalles de confiance à 95\%. Egalement, la fraction étiologique du risque a été calculée afin de quantifier, en pourcentage, la contribution du facteur de risque étudié dans la survenue d'IN ou de décès. L'analyse multifactorielle a été réalisée en utilisant la méthode de régression logistique binaire.

\section{Résultats}

\section{Description de la population d'étude}

Parmi les 335 patients hospitalisés durant la période d'étude, 305 avaient une durée de séjour hospitalier supérieure ou égale à 48 heures.

La distribution de la population selon le sexe montre une très nette prédominance masculine avec un sexe ratio de 3,2. L'âge moyen était de $42,5 \pm 18,7$ ans avec une étendue de 74 ans (minimum : 14 ans, maximum : 88 ans). Les hommes étaient significativement moins âgés que les femmes[ $[40,9 \pm 18,5$ ans contre $46,5 \pm 19,8$ ans, $(p<0,05)]$.
La notion d'antibiothérapie à l'admission était retrouvée chez 298 patients hospitalisés, soit $97,7 \%$ des cas.

Deux patients seulement présentaient une immunodépression documentée avec un taux de polynucléaires circulants inférieur à 500 éléments $/ \mathrm{mm} 3$.

La fréquence des décès en réanimation était estimée à $62,8 \%$.

\section{Description des infections associées aux soins}

Parmi les 305 patients, 73(cas) avaient présenté,au moins, une IN, soit un taux de patients infectés de $23,9 \%$. Parmi ces derniers, 99 IN ont été documentées, correspondant à un taux d'incidence égal à $32,5 \%$.

Le tableau 1 expose les résultats de l'analyse unifactorielle des facteurs de risque liés à la survenue d'IN, toutes localisations confondues. Les facteurs significativement associés à la survenue d'IN étaient : le sexe masculin, un âge $\leq 40$ ans, la présence de traumatisme,quel que soit son type, et la provenance des malades de l'extérieur de l'EHUO.

De même, l'allongement des durées d'exposition aux différents dispositifs invasifs était significativement associé à la survenue d'IN, comme par exemple une durée d'intubation $>8$ jours, une durée de sondage urinaire $>9$ jours et une durée de CVC $>8$ jours.

Le tableau 2 expose les résultats de l'analyse multifactorielle des facteurs de risque liés à la survenue d'IN. Le sexe masculin et une durée de CVC > 8 jours étaient significativement associés à la survenue d'IN.

Le tableau 3 expose les résultats de l'analyse unifactorielle des principaux facteurs pronostiques associés à la survenue de décès. La survenue de décès était significativement associée aux trois facteurs pronostiques suivants : âge $>65$ ans, exposition à l'intubation et présence de CVC.

Ces mêmes facteurs étaient identifiés par régression logistique binaire (tableau 4).

Cependant, la survenue de décès n'était pas associée à la présence d'IN, et ce, quelle que soit sa localisation. 
Tableau 1 - Etude des facteurs de risque liés à la survenue d'IN, toutes localisations confondues, en analyse unifactorielle $(n=305)$

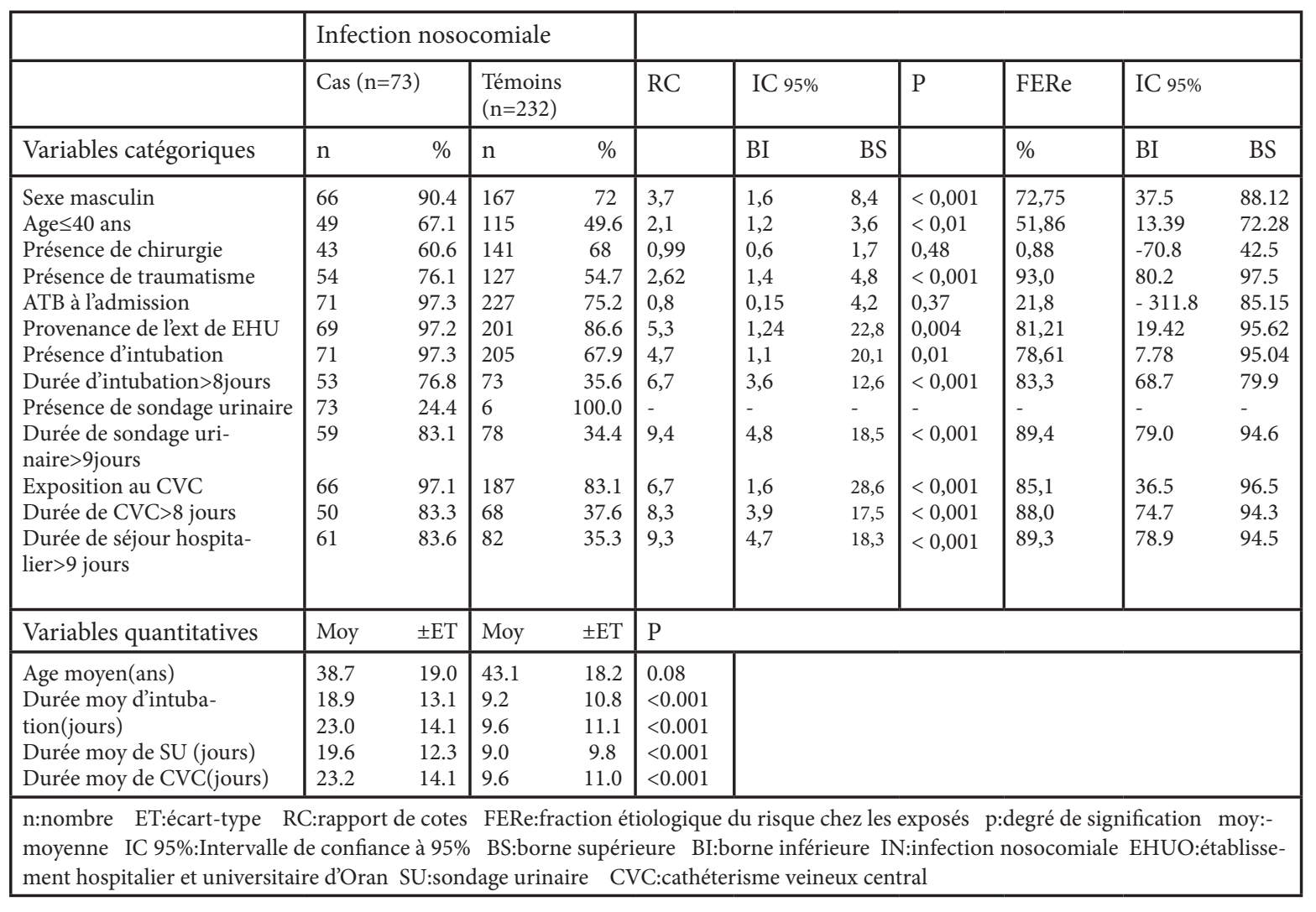

Tableau 2 . Etude des facteurs de risque d'IN en analyse multifactorielle $(n=305)$

\begin{tabular}{|l|l|l|l|l|c|}
\hline Covariables & $\beta$ & RCa & \multicolumn{4}{|c|}{ IC 95\% } & $p$ \\
\hline & & \multicolumn{4}{|c|}{ BI BS } \\
Sexe masculin & 1,15 & 3,16 & 1,11 & 9,04 & 0,03 \\
\hline $\begin{array}{l}\text { Durée de séjour }>9 \\
\text { jours }\end{array}$ & 0,86 & 2,37 & 0,7 & 7,87 & 0,16 \\
\hline $\begin{array}{l}\text { Durée de CVC }>8 \\
\text { jours }\end{array}$ & 1,56 & 4,7 & 1,4 & 16,4 & 0,01 \\
\hline $\begin{array}{l}\text { Durée d'intubation }> \\
8 \text { jours }\end{array}$ & $-0,1$ & 0,9 & $0,97 \quad 4,37$ & 0,84 \\
\hline Constante & $-3,49$ & 0,003 & - & - & 0,03 \\
\hline $\begin{array}{l}\text { B : coefficient } ß \text { RCa: rapport de cotes ajusté IC à 95\% : intervalle de } \\
\text { Confiance à 95\% BI : borne inférieure BS : borne supérieure }\end{array}$ \\
\hline
\end{tabular}


Tableau 3 -Etude des principaux facteurs pronostiques associés à la survenue de décès en analyse unifactorielle $(n=301)$

\begin{tabular}{|c|c|c|c|c|c|c|c|c|c|c|c|}
\hline \multirow[b]{3}{*}{ Variables catégoriques } & \multicolumn{4}{|c|}{ Décès* } & \multirow{3}{*}{$\mathrm{RC}$} & \multirow{2}{*}{\multicolumn{2}{|c|}{ IC $95 \%$}} & \multirow{3}{*}{$\mathrm{P}$} & \multirow{3}{*}{$\begin{array}{l}\text { FERe } \\
\%\end{array}$} & \multirow{2}{*}{\multicolumn{2}{|c|}{ IC 95\% }} \\
\hline & \multicolumn{2}{|c|}{ Cas $(n=189)$} & \multicolumn{2}{|c|}{ Témoins ( $\mathrm{n}=112$ ) } & & & & & & & \\
\hline & $\mathrm{n}$ & $\%$ & $n$ & $\%$ & & $\mathrm{BI}$ & BS & & & BI & BS \\
\hline $\begin{array}{l}\text { Sexe masculin } \\
\text { Age>65 ans } \\
\text { Présence de chirurgie } \\
\text { Présence de traumatisme } \\
\text { ATB à l'admission } \\
\text { Provenance de l'ext de EHU } \\
\text { Présence d'intubation } \\
\text { Présence de sondage urinaire } \\
\text { Présence de CVC } \\
\text { Survenue d'IN } \\
\text { Survenue de pneumopathie } \\
\text { Survenue d'IU } \\
\text { Survenue de bactériémie } \\
\text { Survenue d'ILC }\end{array}$ & $\begin{array}{l}146 \\
32 \\
73 \\
115 \\
187 \\
166 \\
183 \\
187 \\
167 \\
49 \\
43 \\
4 \\
12 \\
7\end{array}$ & $\begin{array}{l}77,2 \\
16,9 \\
38,6 \\
60,8 \\
98,9 \\
87,8 \\
96,8 \\
98,9 \\
91,8 \\
25,9 \\
22,8 \\
2,11 \\
6,35 \\
3,70\end{array}$ & \begin{tabular}{|l|}
83 \\
8 \\
48 \\
64 \\
107 \\
103 \\
89 \\
108 \\
82 \\
21 \\
17 \\
5 \\
4 \\
4 \\
\end{tabular} & $\begin{array}{l}74,1 \\
7,1 \\
42,9 \\
57,1 \\
95,5 \\
84,4 \\
79,5 \\
96,4 \\
76,6 \\
18,8 \\
15,2 \\
4,46 \\
3,57 \\
3,57\end{array}$ & \begin{tabular}{|l}
1,19 \\
2,65 \\
0,84 \\
1,17 \\
4,37 \\
0,63 \\
7,82 \\
3,46 \\
3,39 \\
1,52 \\
1,64 \\
0,46 \\
1,83 \\
1,04
\end{tabular} & $\begin{array}{l}0,69 \\
1,18 \\
0,52 \\
0,72 \\
0,84 \\
0,28 \\
3,09 \\
0,62 \\
1.69 \\
0,85 \\
0,88 \\
0,12 \\
0,57 \\
0,29\end{array}$ & $\begin{array}{l}2,04 \\
6,0 \\
1,35 \\
1,87 \\
22,9 \\
1,41 \\
20,05 \\
19,22 \\
6,78 \\
2,69 \\
3,05 \\
1,76 \\
5,81 \\
3,6\end{array}$ & $\begin{array}{l}0,54 \\
<0,01 \\
0,24 \\
0,52 \\
0,13 \\
0,27 \\
<0,001 \\
0,17 \\
<0,001 \\
0,16 \\
0,059 \\
0,13 \\
0,15 \\
0,48\end{array}$ & $\begin{array}{l}15,7 \\
62,3 \\
16,1 \\
14,2 \\
77,1 \\
36,9 \\
87,3 \\
71,12 \\
70,54 \\
34,07 \\
39,24 \\
53,73 \\
45,37 \\
3,70\end{array}$ & \begin{tabular}{|l}
$-45,02$ \\
14,88 \\
$-34,95$ \\
$-37,9$ \\
$-19,99$ \\
$-41,6$ \\
67,73 \\
$-60,26$ \\
41,12 \\
$-17,21$ \\
$-12,73$ \\
$-76,02$ \\
$-73,66$ \\
$-100,0$
\end{tabular} & $\begin{array}{l}51,0 \\
83,27 \\
47,83 \\
46,63 \\
95,63 \\
71,91 \\
95,01 \\
94,8 \\
85,26 \\
62,91 \\
67,25 \\
87,84 \\
82,82 \\
72,45\end{array}$ \\
\hline Variables quantitatives & Moy & $\pm \mathrm{ET}$ & Moy & $\pm \mathrm{ET}$ & $\mathrm{p}$ & & & & & & \\
\hline $\begin{array}{l}\text { Age moyen(ans) } \\
\text { Durée moy d'intuba- } \\
\text { tion(jours) } \\
\text { Durée moy de SU (jours) } \\
\text { Durée moy de CVC(jours) } \\
\text { Durée moy de séjour(jours) }\end{array}$ & $\begin{array}{l}45,1 \\
11,7 \\
12,4 \\
11,6 \\
12,5\end{array}$ & $\begin{array}{l}19,5 \\
11,6 \\
12,3 \\
10,8 \\
12,6\end{array}$ & $\begin{array}{l}38,2 \\
10,7 \\
12,9 \\
11,4 \\
12,9\end{array}$ & $\begin{array}{l}17,1 \\
13,0 \\
14,0 \\
12,7 \\
13,9\end{array}$ & $\begin{array}{l}0,001 \\
0,53 \\
0,75 \\
0,89 \\
0,77\end{array}$ & & & & & & \\
\hline $\begin{array}{l}\mathrm{N}: \text { nombre ET : écart-type } \\
\text { moy : moyenne IC à } 95 \%: \text { int } \\
\text { EHUO : établissement hospita } \\
\text { ILC : infection liée au cathétér }\end{array}$ & $\begin{array}{l}\text { RC: R } \\
\text { rvalle c } \\
\text { lier et u } \\
\text { isme ve }\end{array}$ & $\begin{array}{l}\text { te } \\
\text { fian } \\
\text { itair } \\
\text { cent }\end{array}$ & $\begin{array}{l}\text { cotes } \mathrm{F} \\
\text { ce à } 95 \\
\text { e d'Or } \\
\text { ral }{ }^{*} \mathrm{~L}\end{array}$ & $\begin{array}{l}=\text { fract } \\
\text { BS : bc } \\
\text { SU } \\
\text { tut vis- }\end{array}$ & $\begin{array}{l}\text { étiol } \\
\text { e sup } \\
\text { nda } \\
\text { s du }\end{array}$ & $\begin{array}{l}\text { e du r } \\
\text { a } \\
\text { aire } \\
\text { était i }\end{array}$ & $\begin{array}{l}\text { chez le } \\
\text { orne ir } \\
\text { : cath } \\
\text { u che }\end{array}$ & $\begin{array}{l}\text { s exposé } \\
\text { férieur } \\
\text { éter vein } \\
4 \text { patien }\end{array}$ & $\begin{array}{l}\text { deg } \\
\text { IN : } \\
\text { cent }\end{array}$ & $\begin{array}{l}\text { nificat } \\
\text { n noss }\end{array}$ & niale \\
\hline
\end{tabular}

Tableau 4 . Etude des facteurs de risque d'IN en analyse multifactorielle $(n=301)$

\begin{tabular}{|c|c|c|c|c|}
\hline Co-variables & $\beta$ & $\mathrm{RCa}$ & $\begin{array}{l}\text { IC 95\% } \\
\text { BI BS }\end{array}$ & $\mathrm{P}$ \\
\hline Age $>65$ ans & 1.46 & 4.31 & 1.5611 .92 & 0.005 \\
\hline Présence d'intubation & 2.25 & 9.49 & 3.2427 .8 & $<0.001$ \\
\hline Présence de CVC & 0.89 & 2.43 & 1.155 .14 & 0.02 \\
\hline $\begin{array}{l}\text { Survenue de pneumo- } \\
\text { pathie }\end{array}$ & 0.14 & 1.15 & 0.572 .33 & 0.69 \\
\hline $\begin{array}{l}\text { Survenue d'infection } \\
\text { urinaire }\end{array}$ & -0.65 & 0.52 & 0.112 .41 & 0.40 \\
\hline Constante & -2.46 & 0.08 & - & $<0.001$ \\
\hline \multicolumn{5}{|c|}{$\begin{array}{l}\beta: \text { coefficient } \beta, \mathrm{RCa} \text {;rapport de cote ajusté à IC95\%:Intervalle de confiance à } \\
95 \%, \mathrm{BI} \text { :Borne inférieure,BS:Borne supérieure } \\
\text { CVC:cathéterisme veineux central }\end{array}$} \\
\hline
\end{tabular}




\section{Discussion}

Dans la présente étude,l'exposition à certains DMI, comme l'intubation et le CVC, était significativement associée, aussi bien à la survenue d'IN qu'à la survenue de décès en réanimation.

\section{Facteurs de risque}

Les facteurs de risque liés à l'acquisition d'IN étaient de deux types. Le premier représentait les facteurs de risque non modifiables ou marqueurs de risque. Dans ce groupe, le rôle du sexe masculin dans la survenue d'IN était rapporté par plusieurs études [11, 12]. Le deuxième facteur de risque identifié, dans la présente étude,était l'âge $\leq 40$ ans, à l'instar de l'étude de Strassle et al.[13] ayant trouvé qu'un âge jeune était significativement associé à la survenue d'IN.Ceci pourrait s'expliquer par le fait que les patients étaient majoritairement de sexe masculin et souvent admis dans le cadre de polytraumatisme. Cependant, la plupart des études indiquent que le risque d'IN, en réanimation adulte, augmente avec l'âge en raison de la sénescence du système immunitaire $[11,12]$.

La deuxième catégorie de facteurs correspondait aux facteurs de risque modifiables comme l'augmentation des durées d'exposition aux différents dispositifs invasifs, laquelle était significativement associée à la survenue d'IN en réanimation adulte de notre établissement. A titre d'exemple, le risque d'IN était 6 fois plus important chez les sujets ayant une durée d'intubation $>8$ jours avec une fraction étiologique du risque chez les exposés égale à $83,3 \%$. En d'autres termes, $83,3 \%$ des cas d'IN étaient attribués à une durée d'intubation $>8$ jours. De ce fait, l'exposition à la ventilation mécanique devrait faire l'objet d'une évaluation quotidienne par le personnel soignant afin de discuter le maintien ou la levée de l'intubation, et par conséquent réduire indirectement le risque infectieux de PAVM [2]. La même mesure de prévention devrait être appliquée aux autres dispositifs invasifs, à l'instar du CVC et du sondage urinaire, lesquels doivent faire l'objet de réévaluations permanentes[2].

La notion d'antibiothérapie à l'admission était présente chez $97,7 \%$ des patients. En effet, l'administration anarchique et prolongée d'antibiotiques serait responsable de taux élevés de multirésistance bactérienne[14].Le risque d'acquisition de bactéries multirésistantes serait associé également à la présence de comorbidités, ce qui est, souvent, le cas dans les services de réanimation [15].

\section{Facteurs pronostiques}

En ce qui concerne les facteurs pronostiques, la présence d'IN n'était pas associée à la survenue de décès dans cette étude, et ce, quelle que soit sa localisation, malgré une fraction étio logique du risque chez les exposés, estimée à 30,07\%, mais non significative.A l'opposé, le risque de décès en présence d'IN était multiplié par 4 dans l'étude d'Iwuafor[16]. De même, la présence de pneumonie ou de bactériémie était associée à la survenue de décès dans une étude cohorte multicentrique, ayant concerné 537 unités de soins intensifs, réparties sur 10 pays européens[17].

Par ailleurs, dans cette étude, les expositions à l'intubation $(\mathrm{RCa}=9,49)$ ainsi qu'au CVC $(\mathrm{RCa}=2,43)$ étaient significativement associées à la survenue de décès,à l'instar del'étude de cohorte de Moreno réalisée dans 9 hôpitaux colombiens[18].

\section{Prévention des IN}

En matière de prévention des $\mathbb{I N}$, l'efficacité de la désinfection hygiénique des mains par une solution hydro-alcoolique a été largement démontrée[19]. Cependant, le véritable défi consiste à convaincre le personnel hospitalier à respecter ce geste, pourtant simple et facile, dans ses pratiques de tous les jours[20].De même, l'utilisation de protocoles écrits et le choix de la veine sus claviculaire au lieu de la veine fémorale constituent des pratiques associées significativement à une réduction des ILC en réanimation[16, 21].

Également, la prévention des infections urinaires est basée sur le respect des mesures d'asepsie lors de la mise des sondes urinaires et le changement régulier de ces dernières tous les 5 à 7 jours[16].De plus, l'intérêt de la position semi-assise (30 $45^{\circ}$ ) dans la prévention des PAVM a été démontré dans la revue de la littérature d'Oliveira [22]. Cette position diminuera le passage des secrétions de l'oropharynx aux voies aériennes sous glottiques[23].

De même, la décontamination oropharyngée à base de chlorhexidine $2 \%$ ou d'antibiotiques[24] ainsi que le drainage des secrétions sous glottiques[25], auraient également un impact positif dans la prévention des PAVM. II faut souligner que la réduction des taux d'IN en réanimation est souvent associée à une réduction de la mortalité comme l'a démontré l'étude de Higuera et al. [26].

\section{Points forts et limites de l'étude}

L'étude cas-témoins nichée dans la cohorte des patients hospitalisés en réanimation offre l'avantage de pouvoir suivre l'exposition aux différents facteurs de risque, depuis l'hospitalisation jusqu'à la survenue de l'évènement étudié. Autrement dit, les chercheurs sont sûrs que l'exposition avait précédé la survenue d'IN ou de décès. Également, l'analyse multifactorielle présente l'avantage de neutraliser les facteurs confondants pouvant être associés aussi bien à la survenue d'IN qu'à la survenue de décès en même temps.

En ce qui concerne les points faibles, il faut souligner que la charge de travail n'a pas fait l'objet d'investigation dans cette étude. En effet, plusieurs travaux avaient démontré que le risque d'IN était associé à une charge de travail importante, ainsi qu'au syndrome d'épuisement professionnel $[27,28]$. 
A contrario, le niveau d'expérience professionnelle et un ratio soignant soigné élevé, étaient inversement proportionnels au risque d'IN[28-30]. Ces facteurs devraient faire l'objet d'une évaluation particulière via la réalisation périodique d'audits permettant de mesurer l'écart entre ce qui est fait et ce qui devrait l'être.

\section{Conclusion}

L'allongement de la durée d'exposition aux différents dispositifs invasifs représentait le principal facteur de risque lié à la survenue d'IN en réanimation. De même, la survenue de décès était principalement associée à un âge $>65$ ans, ainsi qu'à la présence de CVC ou d'intubation. Cependant, aucun lien significatif entre l'IN et la survenue de décès n'a été mis en évidence. De ce fait, la réalisation d'études incluant d'autres paramètres spécifiques, liés essentiellement à certains comportements humains, pourrait apporter des informations complémentaires sur d'éventuels facteurs pouvant jouer un rôle prégnant dans la survenue d'IN ou de décès en réanimation.

\section{Conflits d'intérêt}

Les auteurs déclarent n'avoir aucun conflit d'intérêt.

\section{Références bibliographiques}

[1] Rosenthal VD, Al-Abdely HM, El-Kholy AA,et al. International Nosocomial Infection Control Consortium report, data summary of 50 countries for 2010-2015: Device-associated module. Am J Infect Control. 2016;44(12):1495-504.

[2] Espinasse F, Page B, Cottard-Boulle B. Risques infectieux associés aux dispositifs médicaux invasifs. Revue francophone des laboratoires. $2010 ;(426): 51-63$

[3]Lepape A, Machut A, Savey A. Réseau national Réa-Raisin de surveillance des infections acquises en réanimation adulte-Méthodes et principaux résultats. Méd Intensive Réa. 2018;27(3):197-203.

[4]Melsen WG, Rovers MM, Bonten MJ. Ventilator-associated pneumonia and mortality: a systematic review of observational studies. Crit Care Med. 2009;37(10):2709-18.

[5]Van der Kooi TI, de Boer AS, Manniën J,et al. Incidence and risk factors of device-associated infections and associated mortality at the intensive care in the Dutch surveillance system. Intensive Care Med. 2007;33(2):271

[6]Clec'h C, Schwebel C, Français A,et al. Does catheter-associated urinary tract infection increase mortality in critically ill patients? Infect Control HospEpidemiol. 2007;28(12):1367-73.
[7]Siempos II, Kopterides P, Tsangaris I, et al. Impact of catheter-related bloodstream infections on the mortality of critically ill patients: a meta-analysis. Crit Care Med. 2009;37(7):2283-9.

[8]Horan TC, Andrus M, Dudeck MA. CDC/NHSN Surveillance definition of health care-associated infection and criteria for specific types of infections in the acute care setting. Am J Infect Control. 2008;36(5):309-32.

[9]Kelsey JL, Whittemore AS, Evans AS, et al. Methods in observational epidemiology: Monographs in Epidemiology and Biostatistics; 1996 (Table 12-15).

[10]Merzougui L, Barhoumi T, Guizani T,et al. Les infections nosocomiales en milieu de réanimation : incidence annuelle et aspects cliniques au Service de Réanimation Polyvalente, Kairouan, Tunisie, 2014. Pan Afr Med J. 2018;30.

[11]Wang R-F, Shen S-H, Yen A-F, et al. Risk factors for incidence and case-fatality rates of healthcare-associated infections: a 20-year follow-up of a hospital-based cohort. Epidemiol Infect. 2016;144(1):198206.

[12]Packer SJ, Cairns S, Robertson C, et al. Determining the effect of social deprivation on the prevalence of healthcare-associated infections in acute hospitals: a multivariate analysis of a linked data set. J Hosp Infect. 2015;91(4):351-7.

[13]Strassle PD, Williams FN, Weber DJ, et al. Risk Factors for Health6] Clec'h C, Schwebel C, Français A,et al. Does catheter-associated urinary tract infection increase mortality in critically ill patients? Infect Control HospEpidemiol. 2007;28(12):1367-73.

[14]Ramsamy Y, Hardcastle TC, Muckart DJ. Surviving sepsis in the intensive care unit: The challenge of antimicrobial resistance and the trauma patient. World J Surg. 2017;41(5):1165-9 [15]Harris AD, Pineles L, Anderson $\mathrm{D}$, et al. Which comorbid conditions should we be analyzing as risk factors for healthcare-associated infections? Infect Control HospEpidemiol. 2017;38(4):449-54.

[16]Iwua for AA, Ogunsola FT, Oladele RO, et al. Incidence, clinical outcome and risk factors of intensive care unit infections in the la gosuniversity teaching hospital (LUTH), Lagos, Nigeria. PLoS One. 2016;11(10):e0165242.

[17]Lambert M-L, Suetens C, Savey A, et al. Clinical outcomes of health-care-associated infections and antimicrobial resistance in patients admitted to European intensive-care units: a cohort study. Lancet infect Dis. 2011;11(1):30-8.

[18]Moreno CÁ, Rosenthal VD, Olarte N, et al. Device-associated infection rate and mortality in intensive care units of 9colombianhospitals: findings of the international nosocomial infection control consortium. Infect Control HospEpidemiol. 2006;27(4):349-56.

[19]Pittet D, Allegranzi B, Sax H, et al. Evidence-based model for hand transmission during patient care and the role of improved practices. Lancet infect Dis. 2006;6(10):641-52.

[20]European Centre for Disease Prevention and Control: Annual epidemiological report on communicable diseases in europe 2008. Stockholm, europeancentre for disease prevention and control, 2008. 
[21]Lerebours E, Stetiou M, Armengol-Debeir L. Infection liée à la voieveineusecentrale. Prévention et traitement. Nutrition Clinique et Métabolisme. 2013;27(4):212-7.

[22]Oliveira J, Zagalo C, Cavaco-Silva P. Prevention of ventilator-associatedpneumonia. Rev Port Pneumol. 2014;20(3):152-61.

[23]Voiriot $G$, Mourvilier B, Wolff $M$, et al. Impact de la position du patient sur le risque de pneumonie acquise sous ventilation mécanique. Réanimation. 2013;22(3):265-71.

[24]Seguin P, Launey Y, Nesseler N, et al. Soins de bouche et pneumonies acquises sous ventilation mécaniqueOral care and ventilator-associatedpneumonia. Réanimation. 2013;22(3):238-44.

[25]Lachérade J-C. Drainage des sécrétions sous-glottiques et pneumonies acquises sous ventilation mécaniqueSubglotticsecretions drainage and ventilator-associatedpneumonia. Réanimation. 2013;22(3):257-64.

[26]Higuera F, Rosenthal VD, Duarte P, et al. The effect of process control on the incidence of central venous catheter-associated bloodstream infections and mortality in intensive care units in Mexico. Crit Care Med. 2005;33(9):2022-7.

[27]Daud-Gallotti RM, Costa SF, Guimarães T, et al. Nursing workload as a risk factor for healthcare associated infections in ICU: a prospective study. PLoS One. 2012;7(12):e52342.

[28]Cimiotti JP, Aiken LH, Sloane DM, et al. Nurse staffing, burnout, and health care-associated infection. Am J Infect Control. 2012;40(6):486-90.

[29]Cho S-H, Ketefian S, Barkauskas VH, et al. The effects of nurse staffing on adverse events, morbidity, mortality, and medical costs. Nurs Res. 2003;52(2):71-9.

[30]Hugonnet S, Chevrolet J-C, Pittet D. The effect of workload on infection risk in critically ill patients. Crit Care Med. 2007;35(1):76-81. 
